ENCYCLOPEDDIE Encyclopédie berbère

BERBERE

9 | 1991

9 | Baal - Ben Yasla

\title{
Ben Barour
}

(Pierres de)

\section{J. Maurel et Ph. Lefranc}

\section{OpenEdition}

Journals

Édition électronique

URL : http://journals.openedition.org/encyclopedieberbere/1667

DOI : $10.4000 /$ encyclopedieberbere.1667

ISSN : 2262-7197

\section{Éditeur}

Peeters Publishers

\section{Édition imprimée}

Date de publication : 1 avril 1991

Pagination : 1436-1441

ISBN : 2-85744-509-1

ISSN : 1015-7344

\section{Référence électronique}

J. Maurel et Ph. Lefranc, «Ben Barour », Encyclopédie berbère [En ligne], 9 | 1991, document B57, mis en ligne le 01 avril 2013, consulté le 25 septembre 2020. URL : http://journals.openedition.org/ encyclopedieberbere/1667 ; DOI : https://doi.org/10.4000/encyclopedieberbere.1667

Ce document a été généré automatiquement le 25 septembre 2020.

(c) Tous droits réservés 


\title{
Ben Barour
}

(Pierres de)

\author{
J. Maurel et Ph. Lefranc
}

1 Les Pierres à gorge sahariennes ou Pierres de Ben Barour sont des pierres allongées et volumineuses, munies sensiblement à égale distance de leurs deux extrémités, d'une gorge de section semi-circulaire qui en fait le tour à la manière d'une ceinture sur un corps. Elles sont généralement parallélépipédiques, plus rarement sybcylindriques ou ovoïdes; leurs dimensions sont variables. La plus grande actuellement connue est parallélépipédique et provient de la région d'Ouargla ; longue de $87 \mathrm{~cm}$, large de 23 à 43 $\mathrm{cm}$, épaisse de $20 \mathrm{~cm}$, elle doit peser une centaine de $\mathrm{kg}$. La plus petite est ovoïde et provient d'Edrichinga dans le Borkou; haute de $19 \mathrm{~cm}$, large de 17 au centre, elle pèse $7,4 \mathrm{~kg}$. En moyenne, les pierres à gorge mesurent de 40 à $60 \mathrm{~cm}$ de longueur, de 20 à 30 $\mathrm{cm}$ de largeur et d'épaisseur et pèsent de 30 à $50 \mathrm{~kg}$. Par leurs dimensions et par leur poids qui excluent la possibilité d'un emploi manuel, elles se différencient des outils à rainure ou à gorge : masses, broyeurs et casse-tête dont les formes sont voisines mais qui, destinés à être tenus à la main ou à être emmanchés, sont toujours plus petits et plus légers.

2 Façonnées dans des roches d'origine strictement locale, les pierres à gorge présentent, suivant les régions, une grande diversité de nature lithologique; ce sont tantôt des quartzites du Primaire, tantôt des grès du Primaire ou du Crétacé, tantôt des calcaires appartenant aux différents étages du Crétacé supérieur. Certaines sont des blocs naturels mais d'autres, avant d'être pourvus d'une gorge, ont subi une taille sommaire qui a régularisé la forme, plus ou moins bien arrondi les extrémités, parfois aplani les faces. Le travail a été effectué par martelage ou piquetage, avec des outils de pierre, à l'exclusion, semble-t-il, de tout outil en métal. La gorge est annulaire à une seule exception près (elle est hélicoïdale sur une pierre du Tademaït central). Large de 2 à 10 $\mathrm{cm}$ ( 5 en moyenne), profonde de 0,5 à $4,5 \mathrm{~cm}$ ( 2 à 2,5 en moyenne), elle a été obtenue par un piquetage plus ou moins méticuleux; elle ne porte qu'exceptionnellement des traces de polissage. Dans plusieurs cas de blocs assez plats, ou de blocs parallélépipédiques à arêtes saillantes, on peut n'observer que de profondes encoches sur les arêtes au lieu d'une gorge complète. 
3 La plupart des pierres à gorge n'offrent aucune trace de travail intentionnel autre qu'une mise en forme sommaire et le creusement d'une gorge. Cependant, quelquesunes, et, par exemple, celle découverte dans une cendrière capsienne de Dra-Mta-elMa-el-Abiod, au Sud de Tébessa, présentent des traits incisés, isolés ou groupés, dont la signification nous échappe. La petite pierre d'Edrichinga, à base plane, est ornée d'une double rainure descendant $\mathrm{du}$ sommet vers la gorge centrale et de cercles concentriques incomplets; un autre, provenant probablement de Tabelbalet et conservée au Musée saharien d'Ouargla, est gravée, à l'une de ses extrémités, d'un cercle pointé à 5 rayons inégaux.

4 Il semble que le voyageur Paul Soleillet (1887) soit le premier auteur qui ait signalé l'existence des pierres à gorge sahariennes et rapporté la légende qui les concerne. Par la suite, à quelques variantes près, cette même légende a été recueillie, en divers points du Sahara central, par l'explorateur F. Foureau, le colonel G. Carbillet, le commandant P. Duclos, le lieutenant R. Bureau, le commandant A. Cauneille, le préhistorien H.-J. Hugot; en 1967, le géologue J.-Ph. Lefranc a pu constater qu'elle était encore bien vivante chez les nomades merabtines d'In-Salah. Le récit le plus circonstancié est celui donné par le lieutenant R. Bureau (1955) qui le tenait d'un nomade né auprès du puits de l'oued Tislaouine. La voici :

5 «Il existait autrefois un Arabe nommé Bârour (Ben Barour, disent Foureau et Carbillet) qui savait parfaitement se diriger au Sahara, d'Est en Ouest et du Nord au Sud, rien qu'en observant les étoiles. Il avait conçu le projet de tracer un peu partout de ces voies chamelières qui matérialiseraient, de jour, les directions et seraient d'un précieux secours à ceux qui ne jouissaient pas des mêmes facultés. Pour cela, il utilisait une grosse pierre de forme oblongue qu'il creusait en son milieu et sur tout le pourtour d'une gorge assez peu profonde. Dans cette gorge, il faisait passer une corde (une chaîne, dit Foureau) qu'il attachait par l'autre extrémité au cou de sa monture. Et il allait ainsi, de Ghardaïa à In-Salah et d'Aoulef à Ghadamès. Quand une pierre devenait trop usée, il l'abandonnait et la remplaçait par une nouvelle qu'il avait toujours en réserve sur son chameau. C'est pour cela que ces pierres sont nombreuses, disséminées au Sahara.

«Mais, comme tous les gens célèbres, Bârour a une histoire. Un devin (un marabout, dit Carbillet) lui avait prédit qu'il mourrait un jour, mordu par son chameau garah (c'est-àdire par son chameau pourvu de sa denture d'adulte et présentant sur toutes ses dents une table d'abrasion laissant apparaitre l'ivoire, ce qui se produit vers l'âge de 8 ans). Pour tenter de tromper le sort, il se servait donc de jeunes chameaux qu'il échangeait avant l'âge fatidique. Mais, devenu vieux, il négligea cette prudence et s'aperçut soudain horrifié, que sa bête avait mis les dents du garah. Il s'enfuit, mais l'animal plus agile le rattrapa avant qu'il eût atteint la colline sur laquelle il espérait se réfugier. C'est là qu'il mourut comme le lui avait fixé le devin. Et voilà pourquoi, quelque part, entre El Goléa et Ouargla, il existe une gara que d'aucuns appellent " garet el Bârour » au pied de laquelle est une tombe. Près de cette tombe, est une pierre de Bârour. D'après la version entendue par H.-J. Hugot dans la région d'Aoulef, Ben Barour devait quotidiennement limer les dents de sa chamelle; il omit de le faire un jour et il eut le cou coupé par elle. Soleillet fournit, sur l'époque où vivait Ben Barour, un renseignement moins vague que les autres auteurs ; c'était « à une époque très reculée, au moment où le Touat n'était pas encore habité ». La garet Ben Barour est une butte isolée qui se dresse au bord de la piste chamelière de Ghardaïa à El Goléa par Hassi Djafou, à l'endroit où la piste traverse l'oued Ben Barour ; elle porte effectivement une 
tombe en forme de tumulus circulaire (djeddar) d'un type préislamique ; à proximité, J.Ph. Lefranc a dénombré 11 pierres à gorge et remarqué des blocs bruts, présentant un étranglement en leur milieu, permettant de les utiliser comme pierres à gorge sans taille préalable.

7 Bien que Foureau (1905) ait rapporté en avoir vu lui-même « un très grand nombre ", il n'est que rarement question des pierres à gorge dans les travaux relatifs à l'archéologie ou à l'ethnographie du Sahara publiés au cours des deux premiers tiers du xxe siècle. En 1967, après une enquête de plusieurs années, J. Morel n'en pouvait encore citer que 189, toutes situées dans une aire correspondant au Grand Erg Oriental, au plateau de Tademaiit, au plateau de Tinrhert et à l'Erg Issaouane. Cependant, depuis 1952, le géologue J.-Ph. Lefranc s'est attaché à leur recherche et à leur étude sur le terrain; il a pu porter à plus de 100 le nombre des pierres à gorge actuellement recensées et étendre considérablement leur aire de dispersion. En fait, elles sont disséminées dans toute la zone saharienne depuis la Saoura jusqu'au Fezzan et au Tchad (Borkou). Nombreuses dans le Tademaït (74), particulièrement dans le Tademaït central (26) et dans le Tademaït septentrional (22) où se trouve précisément la garet Ben Barour, elles sont moins fréquentes dans le Touat (9), la Saoura (5), le grand Erg Oriental et la plateau de Tinrhert (9), rares au Tidikelt (1), en Tripolitaine et au Fezzan (3), au Borkou (1), au Tassili N’Ajjer (1) et dans le Sud tébessien (1).

8 A une époque relativement récente, certaines pierres à gorge ont été levées et plantées verticalement comme des bornes (l'une d'elles, déposée depuis au Musée du Bardo à Alger, a servi de repère pendant la construction de la piste de Fort-Flatters à Amguid). Mais, quand elles n'ont pas été déplacées pour un réemploi moderne, elles gisent couchées sur le sol et elles gisent ainsi depuis longtemps puisque la face exposée à l'érosion éolienne est en général beaucoup plus profondément corrodée que les autres faces.

Une seule pierre à gorge est approximativement datée par un contexte archéologique. Elle provient d'une cendrière de Dra-Mta-el-Ma-el-Abiod, à $28 \mathrm{~km}$ au Sud de Tébessa, à la limite septentrionale de la marge steppique qui borde l'Erg Oriental. Longue de 46,8 $\mathrm{cm}$, large et épaisse de 30,6 et 21,6 cm au centre, d'un poids de $36 \mathrm{~kg}$, elle est conservée au Musée d'Hippône à Annaba. Elle a été extraite, par J. Morel, en 1938, d'un niveau capsien terminal qui ne paraissait pas remanié, certainement postérieur aux niveaux profonds du même gisement datés de $5050 \pm 200$ avant J.-C. par le C14, et antérieur peut-être de peu - au Néolithique de tradition capsienne lui-même daté dans la région, à la Table de Jaatcha, en Tunisie, de $3050 \pm 150$ avant J.-C... On peut estimer qu'elle est du iv $v^{e}$ Millénaire avant notre ère, mais rien n'autoriserait à étendre cet âge à l'ensemble des pierres de Ben Barour du Sahara, d'autant qu'elle n'a pas été trouvée dans la zone proprement saharienne, s'il n'y avait d'autres raisons de tenir ces pierres pour très anciennes.

10 En 1957, H. Lhote en a découvert une dans un abri néolithique de Sefar, au Tassili N’Ajjer, abri dit "des masques noirs ", orné de peintures appartenant aux époques bovidienne et post-bovidienne de l'art rupestre saharien ( $\mathrm{IV}^{\mathrm{e}}$ et $\mathrm{III}^{\mathrm{e}}$ Millénaires avant J.C). C'est un bloc long de $36 \mathrm{~cm}$, large de 19 et épais de 16 au centre, d'un poids approximatif de $15 \mathrm{~kg}$; il est muni d'une gorge incomplète et qui présente un polissage superficiel. Cette pierre, qui est la seule connue au Tassili et qui a été déposée au Musée de l'Homme à Paris, reposait sur le sol de l'abri et n'était accompagnée d'aucun autre 
objet témoignant d'une action humaine ; il est possible, mais non certain, qu'elle soit contemporaine des peintures.

11 Aucun contexte préhistorique, archéologique ou ethnologique, ne permet de dater les autres pierres de Ben Barour ; cependant, elles donnent l'impression d'être très vieilles, d'une part parce qu'elles paraissent avoir été façonnées avec des outils de pierre, d'autre part parce que leurs surfaces retouchées ont souvent acquis une forte patine: les grès ont pris une teinte brun-noir, les calcaires sont vermiculés et revêtus d'un enduit luisant caractéristique dit «vernis du désert». Les pierres à gorge sont probablement antérieures à l'introduction du métal au Sahara, à fortiori à la date admise pour l'introduction du chameau.

Pierres de Ben Barour et figures rupestres d'après J.-L. Lequellec.

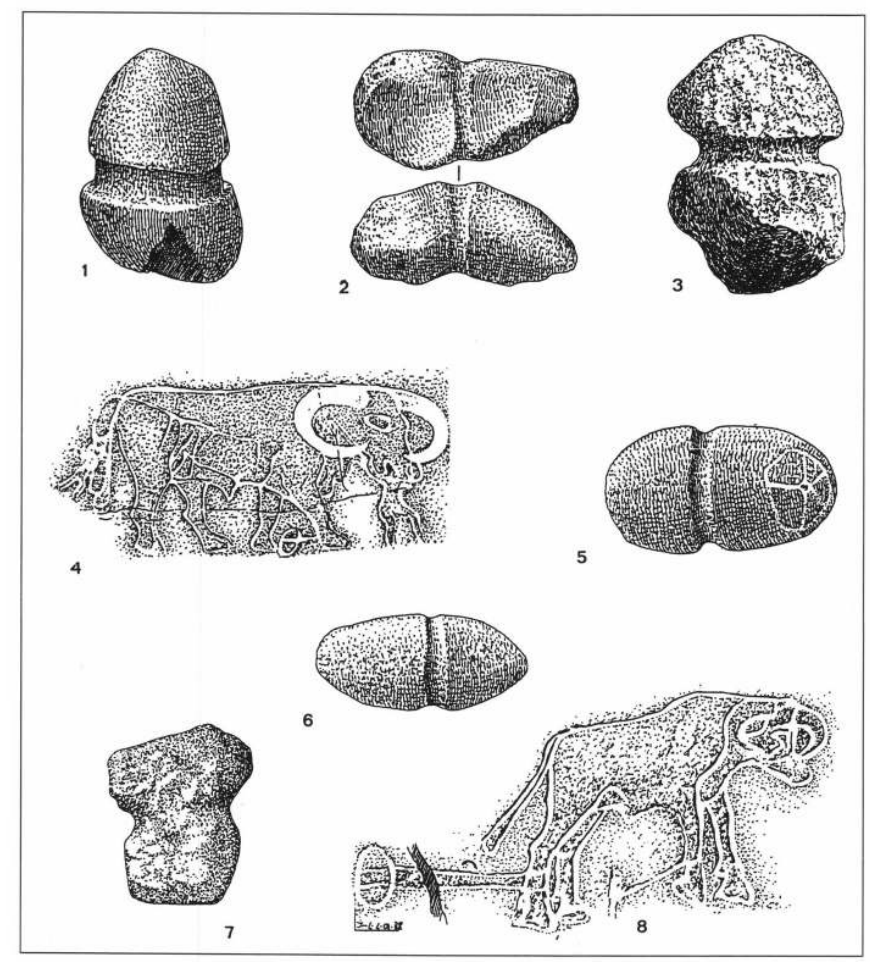

1 et 2. Pierres de Ben Barour d'In Habeter. 3. Pierre à rainure de l'escargotière capsienne de Dra mta elma el-abiod. 4. Gravure de Tel Isaghen (relevé Jelineck : grand bovine retenu par une pierre de Ben Barour). 5, 6 et 7. Pierres de Ben Barour de Tabelbalet, de la région de Mathendûs et des environs de Ghudwa. 8. Gravure rupestre du wadi Geddis ; grand boviné entravé et retenu par une pierre de Ben Barour.

12 S'il est difficile de préciser leur âge, il l'est plus encore de deviner leur destination. La légende de Ben Barour les associe à la mise en place des pistes chamelières, mais il n'est pas vraisemblable qu'elles aient pu servir, soit à les tracer, soit à les baliser. Si elles avaient servi à les tracer, elles présenteraient, au moins sur l'une des faces, une abrasion caractéristique que l'on n'observe pas, la seule forme d'usure apparente et presque générale étant manifestement d'origine éolienne ; de plus, les pierres « usées » auraient dû être abandonnées plus ou moins loin de leur lieu d'extraction alors qu'elles gisent toujours près de celui-ci. Enfin, on peut penser que le remorquage par une attache centrale unique (et non par une attache double proche des extrémités) n'est ni rationnel ni pratique, que le lien de remorquage passé autour de la gorge n'aurait pas 
résisté longtemps au frottement sur le sol et que la trace laissée par la pierre aurait été inefficace sur une hamada, insuffisante sur un reg et trop fugace dans un erg où le premier vent de sable eût tôt fait de l'effacer. Dans l'hypothèse du balisage, la gorge ne se justifie plus, le fait que les pierres ne soient pas dressées mais couchées sur le sol ne s'explique pas; aussi bien, quels services auraient pu rendre des bornes hautes de 19 à $87 \mathrm{~cm}$ (souvent 40 à $60 \mathrm{~cm}$ ) qui, pour être stables, auraient dû être calées à la base par d'autres blocs que l'on ne retrouve pas ou enfoncées dans le sol au moins au tiers de leur hauteur. Les tas de pierres, redjem ou guemaïr, qui permettent aux caravaniers des ergs de retrouver leur route quand les traces laissées par les voyageurs précédents ont disparu, sont beaucoup plus hauts et beaucoup plus volumineux pour être visibles. Il est d'ailleurs remarquable qu'à peine un quart des pierres à gorge aient été découvertes au bord d'une piste chamelière. Les autres sont en relation, non avec un itinéraire menant vers quelque lieu habité, mais avec une zone de pâturages : cuvette limoneuse du genre daïa ou maâder, aire d'épandage alluvial d'un oued, voire même hamada pierreuse où la végétation bien que maigre et diffuse est cependant notablement plus riche qu'aux alentours. Les pierres à gorge sont liées à la végétation spontanée du désert; elles ont été façonnées et abandonnées sur place par des hommes qui menaient une vie de chasseurs et de pasteurs. Or, certaines des aires où on les rencontre ont pratiquement cessé d'être fréquentées par les chasseurs et les pasteurs depuis la fin du dernier épisode humide qu'ait connu le climat saharien ; cet épisode ou " optimum climatique » qui coïncide avec la néolithisation de la zone actuellement aride ne dépasse pas le $\mathrm{III}^{\mathrm{e}}$ Millénaire avant notre ère, la désertification devenant rapide et intense à partir du milieu du Millénaire suivant.

13 La remarquable distribution des pierres de Ben Barour suggère des utilisations possibles qui expliqueraient la présence d'une gorge et les limites de variation de leur poids, poids toujours tel qu'un homme (exceptionnellement deux) puisse les soulever et que cependant leur traction sur le sol soit pénible. Les pierres à gorge ont pu constituer des poids morts efficaces et maniables servant à arrimer des objets (piquets de tente, par exemple), à immobiliser ou à entraver des êtres vivants (ennemi ou condamné, animal domestique, animal sauvage dont l'abattage était différé), à armer des pièges destinés au moyen et gros gibier de plume (autruches) ou de poil (Equidés, Bovinés, Antilopidés, Girafidés...). Les Touaregs utilisent encore trois types de pièges dont les poids-morts sont des pierres qu'un lien enserre.

14 La destination des rares pierres à gorge gravées est plus mystérieuse. Il est tentant d'y voir des objets culturels ou tout au moins chargés d'une signification mystique, ce qu'elles ont peut-être été effectivement. Cependant, l'ethnographie saharienne propose des explications plus réalistes qui d'ailleurs ne sont pas incompatibles avec certaines croyances religieuses ou certaines pratiques magiques. La pierre d'Edrichinga fait d'abord penser à une figuration symbolique pour l'instant indéchiffrable, mais elle présente aussi une remarquable convergence de forme avec le modèle le plus simple des boîtes en peau moulée (tahatint) des Touareg. Le quadruple arceau dont elle est ornée est tout à fait comparable au signe des chasseurs, connu depuis la Nubie jusqu'à l'Atlas saharien et habituellement composé de quatre demi-cercles concentriques. Cette pierre a pu être un lourd casse-tête rituel utilisé par des chasseurs. Celle conservée au Musée de Ouargla est trop pesante pour avoir été un casse-tête ; le cercle pointé et radié qui la décore évoque immédiatement une représentation solaire et l'on n'est pas surpris d'apprendre qu'elle provient probablement de Tabelbalet, haut-lieu de la 
pensée préislamique où ont été trouvées les fameuses «idoles ", bétyles sans bouche, sans nez, sans yeux dont le caractère anthropomorphique n'est plus attesté que par l'ovale du visage. Toutefois, le cercle pointé et radié, dans de nombreuses gravures sahariennes, ne représente sans doute rien de plus qu'un piège à pointes radiaires, équipé ou non d'un lacet relié à un corps mort. La pierre du Musée d'Ouargla était peutêtre un tel corps-mort que la gravure avait enrichi d'une vertu magique.

Ainsi, sous la dénomination commune de pierres à gorge ou pierres de Ben Barour, sont vraisemblablement réunis des objets sahariens différents dont la similitude de forme masque la diversité des utilisations.

Des pierres à gorge sont conservées au Musée de l'Homme à Paris, au Musée du Bardo à Alger, au Musée saharien d'Ouargla, au Musée saharien de Béni-Abbès, au Musée archéologique d'Hippône à Annaba, au laboratoire de géologie de la Faculté des Sciences d'Orsay, ainsi que dans diverses collections privées.

\section{BIBLIOGRAPHIE}

BUREAU Lt. R., « A propos des “Pierres de Barour” ", Bull, de Liaison saharienne, 1954, Alger, t. 6, n 20 (mai 1955) p. 23-24.

CAPOT-REY R., « Découvertes archéologiques au Borkou », Trav. Institut Rech. Sahar., 1958, Université d'Alger, t. 17, p. 203-205, 2 pl.

CAUNEILlE Cdt. A., « Le nomadisme des Zentân (Tripolitaine et Fezzan) », Trav. Institut Rech. Sahar., 1957, Université d'Alger, t. 16 ( $2^{\text {e }}$ sem. 1957), p. 73-99, 6 fig., 2 tabl., bibl.

Duclos Cdt P., in Haardt G.-M., Audouin-Dubreuil L., Le raid Citroën. La première traversée du Sahara en automobile. De Touggourt à Tombouctou par l'Atlantide, 1923, 1 vol., in-16, Plon et Cie édit., Paris, 310 p., 60 photos, 2 cartes, cf. p. 294-301.

FOUREAU F., Documents scientifiques de la mission saharienne Foureau-Lamy. D’Alger au Congo par le Tchad, 1905, Publ. Soc. géog. de Paris, Masson, édit., t. 3, fig., pl., cf. p. 1090.

LE QUELLEC J.-L., « Pierres de Ben Barour et "Radnetzen" au Fezzan (Libye) », L'Anthropologie, t. 94, 1990, p. 115-126.

MOREL J., " Enquête sur les "Pierres de Ben Barour" », Bull, de Liaison saharienne, 1952, Alger, t. 3, n 10 (octobre 1952), p. 8-9.

MOREL J., « Découverte d'une pierre à gorge dans une escargotière capsienne de la région de Tébessa (Algérie). Les pierres à gorge du Sahara oriental », Libyca, 1967, sér. Anthrop. Préhist. Ethn., Alger, t. 15, p. 125-137, 9 fig., bibl. Id. « Les pierres à gorge du Sahara. Inventaire provisoire et essai d'interprétation ", Journal de la Soc. des Africains, t. 52, 1982, p. 68-94.

SAVARY J.-P., «Éléments remarquables du Néolithique saharien », Bull. Soc. Préh. franc., Paris, 1967, t. $64, \mathrm{n}^{\circ} 3$, p. $831-864,8$ fig.

SOLEILLET P., L'Afrique occidentale : Algérie, Mzab, Tildikelt, 1877, 1 vol., in-12, Challamel édit. Paris, 280 p., cf. p. 245-246 (journée du 3 mars 1874). 
INDEX

Mots-clés : Ethnographie, Protohistoire 